\title{
Possible protective role of metformin therapy on colonic polyps in acromegaly: an exploratory cross-sectional study
}

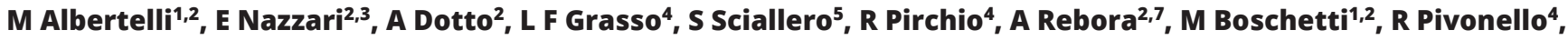 \\ S Ricci Bitti ${ }^{2}$, A A L Colao ${ }^{4,6}$ and D Ferone ${ }^{1,2}$
}

${ }^{1}$ Endocrinology Unit, IRCCS AOU San Martino, Genoa, Italy, ²Endocrinology Unit, Department of Internal Medicine and Medical Specialties (DIMI) and Centre of Excellence for Biomedical Research (CEBR), University of Genoa, Genoa, Italy , ${ }^{3}$ Endocrinology and Diabetology, ASL2 Savonese, Liguria, Italy, ${ }^{4}$ Dipartimento di Medicina Clinica e Chirurgia, Sezione di Endocrinologia, Università Federico II di Napoli, Naples, Italy, ${ }^{5}$ Medical Oncology Unit 1, IRCCS AOU San Martino, Genoa, Italy, ${ }^{6}$ UNESCO Chair for Health Education and Sustainable Development, Federico II University, Naples, Italy, and ${ }^{7}$ SC Diabetology and Metabolic Disease, ASL 3 Genovese, Liguria, Italy

*(M Albertelli and E Nazzari contributed equally to this work)

Correspondence should be addressed to M Albertelli Email manuela.albertelli@unige.it

\begin{abstract}
Context: Colonic polyps occur in $30-40 \%$ of acromegalic patients, increasing the risk of colon carcinoma. Although debated, there is emerging evidence that metformin may play a protective role in diabetic and non-diabetic patients with colonic polyps and its use in chemoprevention is currently explored.

Objective: Evaluate the prevalence of colonic polyps in acromegalic patients treated or not with metformin and explore its possible protective role.

Design: Exploratory cross-sectional study in two tertiary Italian referral centres.

Methods: Out of 153 acromegalic patients, we selected 58 patients (36-82 years; f: 33) who had at least one colonoscopy performed within the first 2 years of diagnosis. Presence of colonic polyps/cancer and related risk factors, current metformin and acetylsalicylic acid intake, disease duration, therapies for acromegaly, hormonal and metabolic parameters were assessed.

Results: An overall prevalence of $36 \%$ polyps was found. Based on the presence of polyps, we identified two groups, comparable for age, BMI, disease duration, glucose, insulin, HOMA-IR, HbA1c, GH and IGF-I levels. Of the patients with polyps (including three adenocarcinomas) only $24 \%$ were treated with metformin vs $57 \%$ of patients without polyps. Multivariate analysis confirmed a significant negative association between colonic polyps and metformin intake (OR: $0.22,95 \% \mathrm{Cl}: 0.06-0.77, P=0.01)$, whereas no significant association was found between polyps and age $(P=0.10)$, overweight/obesity $(P=0.54)$, smoking $(P=0.15)$, acetylsalicylic acid intake $(P=0.99)$, disease duration $(P=0.96)$, somatostatin analogues treatment $(P=0.70)$.

Conclusions: These findings, though deriving from an exploratory study, could suggest a protective role of metformin on the development of colonic polyps in acromegaly, and need to be confirmed in an extended study population.
\end{abstract}

\section{Introduction}

First data showing a marked reduction in cancer incidence in type 2 diabetic patients treated with metformin were published in 2005 (1). To date, although both clinical and

in vitro studies have been providing increasing evidence of the potential protective role of metformin therapy on cancer development $(2,3,4)$, such role is still debated and 
published data are conflicting $(5,6,7,8,9,10,11,12$, $13,14,15,16,17)$. Nowadays, more than 300 randomised clinical trials are ongoing, investigating the anticancer effect of metformin, mostly focusing on tumours for which diabetes and insulin resistance are known risk factors, such as colorectal cancer (18).

Metformin can provide anticancer activity either indirectly, by reducing insulin and insulin-like growth factors levels, or directly, by activating a number of pathways that lead to a reduction of protein transcription, cell growth and proliferation, such as activation of LKB1/ AMPK pathway, induction of cell cycle arrest and/or apoptosis, inhibition of protein synthesis and inhibition of the unfolded protein response (UPR), activation of the immune system and eradication of cancer stem cells (3, $16,17)$.

Among endocrine disorders, acromegaly displays a higher risk of colonic polyps and cancer. As known, this rare condition (prevalence 40-130 cases per million inhabitants) is characterised by chronic growth hormone $(\mathrm{GH})$ and insulin-like growth factor 1 (IGF-I) hypersecretion, generally caused by a pituitary adenoma $(19,20,21)$.

Although the real incidence of cancer in acromegalic patients is widely debated (22), there is general agreement on the increased risk of colonic polyps (up to 30-40\%), with a consequent higher risk of cancer development $(23,24,25,26)$. Recent data from retrospective and prospective studies and metanalyses indicate a 2.4-fold increased risk of colonic adenomas, a 3.7\% prevalence of colorectal cancer with a 2.5-fold higher colon cancerspecific mortality rate in acromegalic patients compared to the general population $(23,24,25,26,27,28)$. In absolute terms, up to $45 \%$ of acromegalic patients might develop colonic polyps. Particularly at risk are male patients with more than 50 years of age, with a duration of disease of more than 5 years and a family history of polyposis $(27,28)$.

Colonic polyps development in acromegalic patients may be influenced first of all by elevated GH and IGF-I levels, stimulating epithelial cell proliferation and inhibit apoptosis, and also by the high prevalence in these patients of impaired glucose tolerance and diabetes, both conditions associated to colorectal cancer development (27, 29). Whereas much has been published about metformin and colorectal cancer (30), no information is available on the effect of metformin on neoplastic colonic lesions development specifically in acromegalic patients.

The main aims of this exploratory study were to confirm the high prevalence of colonic neoplasms in a cohort of acromegalic patients and to explore the possible protective role of metformin in this disease.

\section{Subjects and methods}

In this exploratory cross-sectional study, the clinical archives of two major Italian tertiary pituitary referral centres (Genova and Napoli) were searched to identify all acromegalic patients who had performed at least one colonoscopy within the first 2 years of diagnosis, from 2000 to 2015. In detail, we excluded from the analysis patients who refused the endoscopic examination, patients who performed colonoscopy more than 2 years after the diagnosis of acromegaly, patients who underwent other screening procedures such as virtual colonoscopy or entero-CT and patients who did not perform the examination due to other comorbidities/ frailties of the patient. Moreover, we excluded patients with unreliable examination including colonoscopy early stopped and/or conducted in poor bowel toilet conditions. Furthermore, we considered ineligible those patients who had a negative colonoscopy, performed for other reasons, within 2 years before the diagnosis of acromegaly.

For each patient, the following information was retrieved and filed in a dedicated computerised database: (1) family history or others risk factors for colonic polyps/ cancers; (2) smoking history; (3) time since clinical onset of acromegaly (estimated disease duration); (4) therapies for acromegaly; (5) BMI, biochemical, hormonal and metabolic parameters (GH, IGF-1, glycaemia, insulin, HbA1c, HOMA-IR) at time of colonoscopy; (6) previous or concomitant use of acetylsalicylic acid; (7) current use of metformin at the time of colonoscopy; current use of metformin was defined as continuous metformin therapy for a minimum of six months prior to colonoscopy (metformin therapy was started in these patients only for insulin resistance or diabetes management); (8) colonoscopic findings. According to endoscopic examination we recorded: (a) presence/absence of colonic polyps and cancer; (b) macroscopic characteristics of the lesions found at colonoscopy (sessile or pedunculated), (c) histological characteristics of removed lesions (hyperplastic or adenomatous, tubular or villous, low grade or high grade dysplasia polyps). Patients were divided into two groups based on the presence (=cases, namely 'polyp') or absence (=controls, namely 'no polyp') of colonic neoplasia. Approval from the Medical Ethical Committee of both centres, as well as patients' informed 
consent to use the data for research purposes, were obtained.

\section{Statistical analysis}

We performed descriptive statistical analysis of parameters evaluated in the two study-groups; values are expressed as mean \pm S.D.

$T$-test was used to compare the distribution of patient characteristics in two groups, such as age, sex, BMI, disease duration and GH, IGF-I, glycaemia, insulin, HbA1c and HOMA-IR values.

Associations between categorical variables were assessed by two-tailed Fisher test or Chi-square test.

Due to the retrospective study design, the extent of the risk has been calculated and expressed as odds ratio (OR) (95\% CI). Odds ratios (ORs) and the corresponding 95\% CIs were calculated by means of an unconditional logistic regression model, with the case/control status as the dependent variable and the following as covariates: age, sex, overweight/obesity, duration of acromegaly, smoking habit, current/previous SSA therapy, current/ previous use of acetylsalicylic acid, and current/previous use of metformin. The final model was arrived at by means of a stepdown procedure $(<0.1)$ based on the likelihoodratio test between the models with and without each covariate, one at a time.

Significance was defined for $P<0.05$, always considering two-tailed $\mathrm{p}$ values. All statistical analyses were performed using software IBM - SPSS Statistics (version 22).

\section{Results}

Out of 201 acromegalic patients in active follow-up at two centres, 58 met the inclusion criteria for colonoscopy and resulted eligible for the study (33 females; mean age: $57 \pm$ 11.6 years; age range: $36-82$ years). A flow chart shows all acromegalic patients screened during the recruitment period, and the proportion of patients excluded from the final analysis with the reasons of exclusion (Fig. 1). Previous and ongoing treatments for acromegaly at the time of endoscopy are shown in Table 1.

In our series, we found a total of 28 colorectal polyps among the 21 patients, mostly as sessile polyps $(n=16)$. As far as histological findings, we recorded a prevalence of hyperplastic polyps ( $n=13,46 \%$ of the total), followed by adenomatous polyps ( $n=10,36 \%$ of the total; $5 / 10$

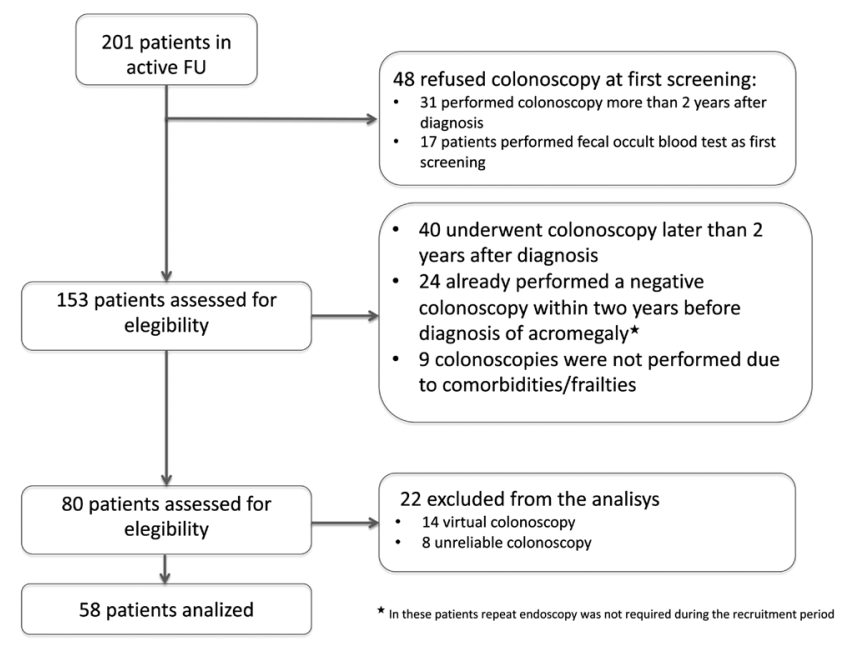

\section{Figure 1}

Flow chart of enrolled patients.

tubular, 3/10 villous and 2/10 tubulo-villous) (Fig. 2), without difference between patients treated or not with metformin. Histological report was unavailable for two lesions (micropolyps not recovered). None of the polyps analysed had a high-degree dysplasia. The endoscopic examination showed the presence of multiple polyps in five patients, including the two subjects with family history of colonic polyps (one with adenocarcinoma). In 3 out of 58 patients $(5.2 \%)$ an adenocarcinoma (with or without accompanying polyps) was found at colonoscopy while in 18 cases (31\%) only a benign polyp was detected, for an overall prevalence of colonic neoplasia of $36.2 \%$.

The characteristics of the 58 patients according to the presence (polyp) or absence (no polyp) of colonic neoplasia are shown in Table 2 . The two groups 'polyp' and 'no polyp' were comparable for several features (Table 2). Cases of polyp were more frequently males (12/21, $57.1 \%)$ than controls whit no polyp $(13 / 37,35 \%)$ but the difference was not statistically significant $(\mathrm{OR}=2.4695 \%$ CI: 0.82-7.37, $P=0.16)$.

However, a striking difference between the two groups was observed in the proportion of patients with diabetes,

Table 1 Previous and ongoing treatments for acromegaly at the time of endoscopy.

\begin{tabular}{lcc}
\hline Therapies & & Number of patients \\
\hline Neurosurgery & 34 \\
Somatostatin analogues (SSA) & 32 \\
SSA + cabergoline & 6 \\
SSA + pegvisomant & 6 \\
Cabergoline & 1 \\
Pegvisomant & 1 \\
\hline
\end{tabular}




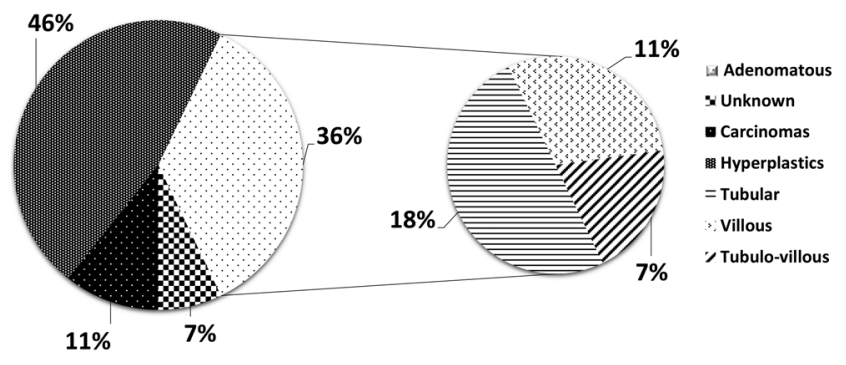

Figure 2

Polyps subdivision according to histotype.

that was present in 6/21 (28.6\%) cases and in 24/37 (64.9\%) controls (OR: 0.22, 95\% CI: 0.07-0.69, $P=0.01$ ). This difference was reflected in the proportion of patients reporting current or previous use of metformin 5/21 (23.8\%) and 21/37 (56.8\%), respectively (OR: 0.24, 95\% CI: $0.07-0.79, P=0.02)$. The duration of metformin therapy changed from a minimum of 6 months to a maximum of 15 years prior to endoscopy, with an average duration of 3 years. The average dose of metformin was $1797 \pm 926$ mg for 'no polyp' and $1100 \pm 540 \mathrm{mg}$ for 'polyp', with no statistically significant difference between the two groups $(P=0.12)$. Four diabetic patients were on only diet-therapy, whereas four were taking other antidiabetic therapies in addition to metformin ( 1 sulfonylurea and 3 insulin). Only one patient among these latter had polyps at colonoscopy, while the others had a negative endoscopic exam.

Univariate analysis confirmed a significant negative association between the presence of polyps and metformin intake. Multivariate analysis (Table 3) as well confirmed a

Table 2 Demographics and patients' characteristics. Data are presented as $n(\%)$ or as mean \pm S.D.

\begin{tabular}{|c|c|c|c|}
\hline Characteristics & Group $1(n=37)$ & Group $2(n=21)$ & $P$ value \\
\hline \multicolumn{4}{|l|}{ Sex, $n(\%)$} \\
\hline Female & $24(64.9)$ & $9(42.9)$ & 0.1 \\
\hline Male & $13(35.1)$ & $12(57.1)$ & \\
\hline Age $(y)$ & $56.2 \pm 2.0$ & $57.6 \pm 2.3$ & 0.67 \\
\hline Disease duration (y) & $12.5 \pm 0.9$ & $13.0 \pm 1.6$ & 0.78 \\
\hline Diabetes, $n(\%)$ & $24(64.9)$ & $6(28.6)$ & 0.01 \\
\hline Smoking, $n(\%)$ & $6(16.2)$ & $6(28.6)$ & 0.26 \\
\hline ASA, $n(\%)$ & $6(16.2)$ & $3(14.3)$ & 0.85 \\
\hline Metformin, $n(\%)$ & $21(56.8)$ & $5(23.8)$ & 0.02 \\
\hline Glycemia (mg/dL) & $112.86 \pm 4.84$ & $103.52 \pm 9.22$ & 0.34 \\
\hline HbA1c (\%) & $6.7 \pm 0.2$ & $6.0 \pm 0.3$ & 0.18 \\
\hline HOMA-IR & $5.1 \pm 1.46$ & $6.54 \pm 3.15$ & 0.64 \\
\hline $\mathrm{BMI}\left(\mathrm{kg} / \mathrm{m}^{2}\right)$ & $28.8 \pm 0.8$ & $29.7 \pm 1.3$ & 0.54 \\
\hline $\mathrm{GH}(\mu \mathrm{g} / \mathrm{L})$ & $10.29 \pm 5.82$ & $5.74 \pm 2.01$ & 0.46 \\
\hline IGF-I,X ULN & $1.80 \pm 1.49$ & $1.88 \pm 1.64$ & 0.86 \\
\hline
\end{tabular}

ASA, acetylsalicylic acid; HbA1c, glycated hemoglobin; IGF-I, Insulin like Growth Factor I; ULN, upper limit of normal. significant negative association between the presence of colonic neoplasia and the use of metformin (OR: 0.22, 95\% CI: $0.06-0.77, P=0.01)$. No association was found between acetylsalicylic acid intake and polyps $(P=0.99)$. In the multivariate analysis, the only two variables with a protective role towards colonic polyps were metformin and female sex (OR: 0.37, 95\% CI: 0.11-1.20, $P=0.09$ ), the latter with a less strong degree. All other variables, age $(P=0.10)$, overweight/obesity $(P=0.54)$, smoking $(P=0.15)$, acetylsalicylic acid $(P=0.99)$, estimated disease duration $(P=0.96)$, somatostatin analogues treatment $(P=0.70)$ were excluded by the model (Table 3$)$.

The degree of protection afforded by metformin appeared to be similar for hyperplastic and adenomatous polyps $(\mathrm{OR}=0.30$ 95\% CI: $0.06-1.58$ and $\mathrm{OR}=0.41 \mathrm{CI}$ : $0.17-0.95$, respectively).

None of the patients with multiple lesions were taking, or had previously taken, metformin.

\section{Discussion and conclusions}

A unique opportunity to study the potential role of metformin as a cancer chemopreventive agent is offered by acromegalic patients, who have a 2.4-fold increased risk of colonic adenomas, a 2.5-fold increased colorectal cancer mortality rate, as compared to general population $(23,24,25,26,27,28,29,30,31,32)$ and often develop type II diabetes (29).

In this exploratory cross-sectional study, the overall prevalence of colonic polyps (36\%) and cancer $(5,2 \%)$ in 58 patients with acromegaly was in line with literature.

Similarly, mean age (over 50 years), estimated disease duration (more than 5 years) at the time of polyps' detection and prevalence of diabetes mellitus type 2 (51.7\%) resulted in line with previously published data (21).

When patients with and without polyps were compared, the presence of diabetes and use of metformin were negatively associated with the presence of polyps, with odds ratios of controls, respectively. Since most diabetic patients (26 out of 30 ) were treated with metformin, and all patients treated with metformin received the drug for insulin resistance or diabetes, it was not possible to evaluate, statistically, which one of these two factors was independently associated with the presence of polyps: as a consequence, only biological plausibility dictated the choice to include metformin use instead of diabetes in multivariate analyses, which fully confirmed the negative association. It is known that the pathophysiology of diabetes facilitates the onset of cancer $(14,15)$. 
Table 3 Univariate and multivariate analysis.

\begin{tabular}{|c|c|c|c|c|}
\hline \multirow[b]{2}{*}{ Risk factors } & \multicolumn{2}{|c|}{ Univariate analysis } & \multicolumn{2}{|c|}{ Multivariate analysis } \\
\hline & Odds ratio $(95 \% \mathrm{Cl})$ & $P$ & Odds ratio $(95 \% \mathrm{Cl})$ & $P$ \\
\hline Age & $2.22(0.37-13.12)$ & 0.37 & $\star \star$ & 0.10 \\
\hline Female Sex & $0.27(0.07-1.07)$ & 0.06 & $0.372(0.11-1.20)$ & 0.09 \\
\hline Overweight/obesity & $0.74(0.11-4.89)$ & 0.78 & $\star \star$ & 0.54 \\
\hline Smoking & $3.83(0.67-21.94)$ & 0.23 & $\star \star$ & 0.15 \\
\hline Disease duration & $0.68(0.22-2.12)$ & 0.72 & ** & 0.96 \\
\hline SSA therapy & $0.92(0.28-3.05)$ & 0.85 & $\star *$ & 0.70 \\
\hline Acetylsalicylic acid & $0.86(0.19-3.87)$ & 0.85 & $\star \star$ & 0.99 \\
\hline Metformin & $0.24(0.07-0.79)$ & 0.02 & $0.224(0.06-0.77)$ & 0.01 \\
\hline
\end{tabular}

Moreover, a recent retrospective study on a large number of acromegalic patients, showed that diabetic acromegalic patients may develop extra pituitary tumours and malignant neoplasms respectively twice and three times more frequently than those without diabetes (29). As stated before, in our series the vast majority of diabetic patients had a negative colonoscopy. According to this observation, patients with dual risk factors for polyps' development, such as diabetes and acromegaly, displayed a lower polyps prevalence compared with non-diabetic acromegalic patients, differently from the expected outcome. Since the only significant difference between the two groups was metformin intake, this finding could suggest that metformin therapy can be able to counterbalance the dual risk factor.

Due to the small number of diabetic patients taking other antidiabetic therapies (including insulin), it was not possible to assess the presence of a correlation between the prevalence of polyps and other hypoglycaemic therapies.

In our study population, there is a trend towards a more frequent presence of polyps in the male gender, in line with that has already been suggested in acromegalic subjects and more strongly in the general population (28). Moreover, no significant difference was found between the two groups in GH and IGF-I values. These results confirm those of previous studies, where no correlation was seen between IGF-I levels and polyps detection at first colonoscopy, even though in a study higher IGF-I values were associated with a higher incidence of polyp recurrence at the endoscopic follow-up (23). Notably, mean GH and IGF-I values recorded in our series were higher than values recommended for disease control (33), since many patients performed colonoscopy near the diagnosis.

In our series, no relevant correlation was found between the presence of colonic polyps and overweight/ obesity. The analysis on fasting insulin levels and
HOMA-IR, though limited by the proportion of missing data (due to retrospective nature of this study), failed to show differences between the two groups. To this regard, it must be considered that the role of insulin resistance in colonic polyps development in acromegaly is still debated. Two recent studies could not find any correlation between serum insulin levels and polyps in acromegaly $(34,35)$. In contrast, Colao et al. reported that an increase in fasting insulin levels is associated with an 8.6- to 14.8fold increased risk of colonic adenomas (36).

Medical therapy for acromegaly was not correlated with polyps development (SSA, $P=0.70$ ). Moreover, although published data suggest a protective effect of acetylsalicylic acid on colonic polyps, in our patients we did not find any significant correlation $(P=0.99)$. Similarly, although smoking is considered a risk factor, we failed to find any association between smoking and polyps $(P=0.15)$.

These results suggest that metformin use significantly affected the development of colonic polyps in our cohort of acromegalic patients, making speculate that a protective role of the drug in this setting can be hypothesized.

Colonic polyps development in acromegalic patients can have several causes: not only elevated GH and IGF-I levels and high incidence of impaired glucose tolerance as well as diabetes, but also genetic factors, increased large bowel length and/or altered bowel transit time, altered acid bile secretion, altered local immune response. As a consequence, extrapolation of the anti-tumour effect of metformin, observed in this specific setting, to the general population is absolutely not warranted.

We are aware that this study has some limitations, mainly due to its retrospective design (including the use of different hormonal assays, the variable dose and duration of metformin therapy and the lack of a third control group of acromegalic diabetic patients not-exposed to metformin). Conversely, the small sample size, due to the low incidence of acromegaly and the choice to include only 
patients who underwent a reliable colonoscopy within two years of diagnosis, is due to the intent of evaluating a homogeneous population without interfering factors, and to make our cohort representative for only an exploratory study. Colonoscopy represents a screening procedure in patients with acromegaly, especially for patients with long term disease history and those with familiarity for colonic cancer. Indeed, acromegaly is reported among the conditions at high risk of colonic neoplasms for which the dedicated guidelines suggest performing colonoscopy at diagnosis. However, despite these evidences, being colonoscopy a rather invasive technique, still many patients refuse it. Moreover, for similar reasons, other patients were not eligible for the procedure. In this setting, when patients refuse or is not eligible, we may delay it or adopt alternative, although less effective, screening procedures. This is the main reason why we collected the data from $30 \%$ patients of the full cohort.

Keeping in mind these limitations our data suggest a possible protective role of metformin for preventing colon polyps in acromegalic patients. Such finding needs to be further assessed with prospective studies that include groups of non-diabetic acromegalic patients, as well as diabetic subgroups exposed and not-exposed to metformin.

In conclusion, despite these limitations, the study suggests a potential efficacy of metformin in preventing colonic polyps development in acromegalic patients.

This protective effect of metformin, once explored and confirmed in a larger population, possibly in a prospective setting, may open the perspective of a future application of metformin in chemoprevention in acromegaly, as well as in other populations with increased risk of colonic cancer.

\section{Declaration of interest}

All the authors declare that there is no conflict of interest that could be perceived as prejudicing the impartiality of this manuscript.

\section{Funding}

This research was partially supported by the Italian Ministry of Education, Research and University (PRIN 2017Z3N3YC)

\section{Acknowledgements}

The authors acknowledge Prof Paolo Bruzzi (Epidemiology Unit, IRCCS Ospedale Polilinico San Martino, Genova, Italy) for providing an excellent statistical analysis revision.

\section{References}

1 Evans JM, Donnelly LA, Emslie-Smith AM, Alessi DR \& Morris AD.

Metformin and reduced risk of cancer in diabetic patients.

BMJ 2005330 1304-1305. (https://doi.org/10.1136/

bmj.38415.708634.F7)
2 Landman GW, Kleefstra N, van Hateren KJ, Groenier KH, Gans RO \& Bilo HJ. Metformin associated with lower cancer mortality in type 2 diabetes: ZODIAC-16. Diabetes Care 201033 322-326. (https://doi. org/10.2337/dc09-1380)

3 Salani B, Del Rio A, Marini C, Sambuceti G, Cordera R \& Maggi D. Metformin, cancer and glucose metabolism. Endocrine-Related Cancer 201421 R461-R471. (https://doi.org/10.1530/ERC-14-0284)

4 Zhang P, Li H, Tan X, Chen L \& Wang S. Association of metformin use with cancer incidence and mortality: a meta-analysis. Cancer Epidemiology 201337 207-218. (https://doi.org/10.1016/j. canep.2012.12.009)

5 Pollak M. Potential applications for biguanides in oncology. Journal of Clinical Investigation 2013123 3693-3700. (https://doi.org/10.1172/ JCI67232)

6 Kasznicki J, Sliwinska A \& Drzewoski J. Metformin in cancer prevention and therapy. Annals of Translational Medicine 2014257. (https://doi.org/10.3978/j.issn.2305-5839.2014.06.01)

7 Ramjeesingh R, Orr C, Bricks CS, Hopman WM \& Hammad N. A retrospective study on the role of diabetes and metformin in colorectal cancer disease survival. Current Oncology 201623 e116-e122. (https://doi.org/10.3747/co.23.2809)

8 Higurashi T, Hosono K, Takahashi H, Komiya Y, Umezawa S, Sakai E, Uchiyama T, Taniguchi L, Hata Y, Uchiyama S, et al. Metformin for chemoprevention of metachronous colorectal adenoma or polyps in post-polypectomy patients without diabetes: a multicentre doubleblind, placebo-controlled, randomised phase 3 trial. Lancet. Oncology 201617 475-483. (https://doi.org/10.1016/S1470-2045(15)00565-3)

9 Zhang ZJ, Zheng ZJ, Kan H, Song Y, Cui W, Zhao G \& Kip KE. Reduced risk of colorectal cancer with metformin therapy in patients with type 2 diabetes: a meta-analysis. Diabetes Care 201134 2323-2328. (https://doi.org/10.2337/dc11-0512)

10 Smiechowski B, Azoulay L, Yin H, Pollak MN \& Suissa S. The use of metformin and colorectal cancer incidence in patients with type II diabetes mellitus. Cancer Epidemiology, Biomarkers \& Prevention 2013 22 1877-1883. (https://doi.org/10.1158/1055-9965.EPI-13-0196)

11 Cho YH, Ko BM, Kim SH, Myung YS, Choi JH, Han JP, Hong SJ, Jeon SR, Kim HG, Kim JO, et al. Does metformin affect the incidence of colonic polyps and adenomas in patients with type 2 diabetes mellitus? Intestinal Research 201412 139-145. (https://doi. org/10.5217/ir.2014.12.2.139)

12 Fernandez-Fernandez FJ. Antineoplastic potential of metformin in colorectal cancer. European Journal of Internal Medicine 201737 e22. (https://doi.org/10.1016/j.ejim.2016.08.034)

13 Rokkas T \& Portincasa P. Colon neoplasia in patients with type 2 diabetes on metformin: a meta-analysis. European Journal of Internal Medicine 201633 60-66. (https://doi.org/10.1016/j.ejim.2016.05.027)

14 Cheng Y, Chen Y, Zhou C, Shen L, Tu F, Xu J \& Liu C. For colorectal cancer patients with type II diabetes, could metformin improve the survival rate? A meta-analysis. Clinics and Research in Hepatology and Gastroenterology 202044 73-81. (https://doi.org/10.1016/j. clinre.2019.06.009)

15 Dankner R, Agay N, Olmer L, Murad H, Keinan Boker L, Balicer RD $\&$ Freedman LS. Metformin treatment and cancer risk: cox regression analysis, with time-dependent covariates, of 320,000 persons with incident diabetes mellitus. American Journal of Epidemiology 2019188 1794-1800. (https://doi.org/10.1093/aje/kwz157)

16 Saraei P, Asadi I, Kakar MA \& Moradi-Kor N. The beneficial effects of metformin on cancer prevention and therapy: a comprehensive review of recent advances. Cancer Management and Research 201911 3295-3313. (https://doi.org/10.2147/CMAR.S200059)

17 Kourelis TV \& Siegel RD. Metformin and cancer: new applications for an old drug. Medical Oncology 201229 1314-1327. (https://doi. org/10.1007/s12032-011-9846-7)

18 clinicaltrials.gov. Available at: (https://clinicaltrials.gov/).

19 Chanson P, Salenave S \& Kamenicky P. Acromegaly. Handbook of Clinical Neurology 2014124 197-219. (https://doi.org/10.1016/B9780-444-59602-4.00014-9) 
20 Gatto F, Trifirò G, Lapi F, Cocchiara F, Campana C, Dell'Aquila C, Ferrajolo C, Arvigo M, Cricelli C, Giusti M, et al. Epidemiology of acromegaly in Italy: analysis from a large longitudinal primary care database. Endocrine 201861 533-541. (https://doi.org/10.1007/ s12020-018-1630-4)

21 Gadelha MR, Kasuki L, Lim DST \& Fleseriu M. Systemic complications of acromegaly and the impact of the current treatment landscape: an update. Endocrine Reviews 201940 268-332. (https:// doi.org/10.1210/er.2018-00115)

22 Melmed S. Acromegaly and cancer: not a problem? Journal of Clinical Endocrinology \& Metabolism 200186 2929-2934. (https://doi. org/10.1210/jcem.86.7.7635)

23 Terzolo M, Reimondo G, Gasperi M, Cozzi R, Pivonello R, Vitale G, Scillitani A, Attanasio R, Cecconi E, Daffara F, et al. Colonoscopic screening and follow-up in patients with acromegaly: a multicenter study in Italy. Journal of Clinical Endocrinology \& Metabolism 200590 84-90. (https://doi.org/10.1210/jc.2004-0240)

24 Rokkas T, Pistiolas D, Sechopoulos P, Margantinis G \& Koukoulis G. Risk of colorectal neoplasm in patients with acromegaly: a metaanalysis. World Journal of Gastroenterology 200814 3484-3489. (https://doi.org/10.3748/wjg.14.3484)

25 Lois K, Bukowczan J, Perros P, Jones S, Gunn M \& James RA. The role of colonoscopic screening in acromegaly revisited: review of current literature and practice guidelines. Pituitary 201518 568-574. (https:// doi.org/10.1007/s11102-014-0586-5)

26 Gonzalez B, Vargas G, Mendoza V, Nava M, Rojas M \& Mercado M. The prevalence of colonic polyps in patients with acromegaly: a case-control, nested in a cohort colonoscopic study. Endocrine Practice 201723 594-599. (https://doi.org/10.4158/EP161724.OR)

27 Vilar L, Naves LA, Caldato C \& Caldato M. Acromegaly and colorectal cancer. Translational Gastrointestinal Cancer 20154 28-38.

28 Koksal AR, Ergun M, Boga S, Alkim H, Bayram M, Altuntas Y, Ozguven Yilmaz B \& Alkim C. Increased prevalence of colorectal polyp in acromegaly patients: a case-control study. Diagnostic and Therapeutic Endoscopy 20142014 152049. (https://doi. org/10.1155/2014/152049)
29 Cheng S, Gomez K, Serri O, Chik C \& Ezzat S. The role of diabetes in acromegaly associated neoplasia. PLOS ONE $2015 \mathbf{1 0}$ e0127276. (https://doi.org/10.1371/journal.pone.0127276)

30 Kamarudin MNA, Sarker MMR, Zhou JR \& Parhar I. Metformin in colorectal cancer: molecular mechanism, preclinical and clinical aspects. Journal of Experimental \& Clinical Cancer Research 201938 491. (https://doi.org/10.1186/s13046-019-1495-2)

31 Jenkins PJ, Frajese V, Jones AM, Camacho-Hubner C, Lowe DG, Fairclough PD, Chew SL, Grossman AB, Monson JP \&, Besser GM. Insulin-like growth factor I and the development of colorectal neoplasia in acromegaly. Journal of Clinical Endocrinology \& Metabolism 200085 3218-3221. (https://doi.org/10.1210/jcem.85.9.6806)

32 Jenkins PJ \& Besser M. Clinical perspective: acromegaly and cancer: a problem. Journal of Clinical Endocrinology \& Metabolism 200186 2935-2941. (https://doi.org/10.1210/jcem.86.7.7634)

33 Giustina A, Chanson P, Kleinberg D, Bronstein MD, Clemmons DR, Klibanski A, van der Lely AJ, Strasburger CJ, Lamberts SW, Ho KK, et al. Expert consensus document: a consensus on the medical treatment of acromegaly. Nature Reviews. Endocrinology 201410 243-248. (https://doi.org/10.1038/nrendo.2014.21)

34 Lombardi M, Scattina I, Sardella C, Urbani C, Marciano E, Signori S, Ruocco L, Pellegrini G, Martino E \& Bogazzi F. Serum factors associated with precancerous colonic lesions in acromegaly. Journal of Endocrinological Investigation 201336 545-549. (https://doi. org $/ 10.3275 / 8812)$

35 Yamamoto M, Fukuoka H, Iguchi G, Matsumoto R, Takahashi M, Nishizawa H, Suda K, Bando H \& Takahashi Y. The prevalence and associated factors of colorectal neoplasms in acromegaly: a single center based study. Pituitary 201518 343-351. (https://doi. org/10.1007/s11102-014-0580-y)

36 Colao A, Pivonello R, Auriemma RS, Galdiero M, Ferone D, Minuto F, Marzullo P \& Lombardi G. The association of fasting insulin concentrations and colonic neoplasms in acromegaly: a colonoscopybased study in 210 patients. Journal of Clinical Endocrinology \& Metabolism 200792 3854-3860. (https://doi.org/10.1210/ jc.2006-2551)

Received 12 August 2020

Revised version received 7 January 2021

Accepted 13 January 2021 